\title{
Lower-hybrid waves generated by anomalous Doppler resonance in auroral plasmas
}

\author{
R. B I N GH A M ${ }^{1,2}$, R. A. C A I R N S ${ }^{2}$, I. V O R G U L \\ and V. D. SH A P I O O \\ ${ }^{1}$ Rutherford Appleton Laboratory, Chilton,Oxon,OX11 0QX, UK \\ (bob.bingham@stfc.ac.uk) \\ ${ }^{2}$ Department of School of Mathematics and Statistics, University of St Andrews, \\ St Andrews, Fife, Scotland, KY16 9SS \\ ${ }^{3}$ Department of Physics, University of California, San Diego, La Jolla, CA 92091, USA
}

(Received 4 December 2009 and accepted 13 December 2009, first published online 21 January 2010)

\begin{abstract}
This paper describes some aspects of lower-hybrid wave activity in space plasmas. Lower-hybrid waves are particularly important since they can transfer energy efficiently between electrons and ions in a collisionless magnetized plasma. We consider the 'fan' or anomalous Doppler resonance instability driven by energetic electron tails and show that it is responsible for the generation of lower-hybrid waves. We also demonstrate that observations of their intensity are sufficient to drive the modulational instability.
\end{abstract}

It is an honor to write this paper for the special edition of the Journal of Plasma Physics honoring Padma K. Shukla.

There is considerable interest in the role lower-hybrid waves play in plasma heating and acceleration of charged particles in space and astrophysical plasmas. They are also extensively used for ion heating and electron current drive in tokamak plasmas (Karney 1977; Karney and Frisch 1978; Cairns 1991). In space plasmas, lower-hybrid waves are easily generated through a variety of different instabilities driven by the free energy contained in anisotropic ion distributions, such as ion conics, ion ring distributions, cross-field electron currents, energetic electron beams etc. It is well known that intense lower-hybrid waves are responsible for a number of nonlinear effects including parametric instabilities and strong wave collapse (Sotnikov et al. 1978; Robinson 1997) initiated by the modulational instability (Bingham et al. 1991; Shapiro et al. 1995b; Bingham et al. 1996). This type of modulational instability results in large-scale density structures accompanied by intense localized lower-hybrid wave packets. Space plasma observations have conclusively shown the existence of intense wave activity around the lower-hybrid resonance. They have been observed as localized structures in density cavitons and are associated with transversely accelerated ions, as well as parallel electron acceleration and suprathermal tail formation. Lower-hybrid waves have been associated with the acceleration of electrons in collisionless shocks. A key dissipation mechanism in quasi-perpendicular shocks is the ion reflection from the sharp potential rise, which sometimes gives rise to a broad shallow foot in the magnetic profile. Clear evidence of the foot and reflected ions was found at the earth's bow shock by Paschmann 
et al. (1982), and computer simulations of shocks (Lembege and Dawson 1985) also confirm the presence of ion ring distributions. Similar ion ring distributions are found in counter-streaming plasma flows, such as in the mantle of non-magnetized planets (Shapiro et al. 1995a; Bingham et al. 2008) and in cometary atmospheres (Johnstone et al. 1987). They have also been suggested as being responsible for electron heating and acceleration in solar flares (McClements et al. 1990) and supernova remnants, such as PKS1209-52 (Bingham et al. 2004b). In this paper we consider aspects associated with lower-hybrid waves in the aurora. This work is also relevant to the studies of the magnetopause region of the magnetosphere (Trines et al. 2005, 2007), the dayside mantle of non-magnetized planets, such as Venus, Mars and comets (Shapiro et al. 1995a; Bingham et al. 1997; Bingham et al. 1999; Shapiro et al. 1999).

Lower-hybrid waves are a branch of oscillation of magnetized plasmas, especially important for space physics and astrophysics. The oscillations are sufficiently of short wavelength $k c \gg \omega_{p e}$, i.e. wavelengths much less than the electron skin depth $c / \omega_{p e}$, and are therefore mainly electrostatic in nature. They propagate almost across the magnetic field lines, having frequency, $\omega$, between the proton and electron gyro frequencies $\omega_{c p} \ll \omega \ll \omega_{c e}$, where $\omega_{c p}$ is the proton gyro frequency and $\omega_{c e}$ is the electron gyro frequency. Due to this, electrons are strongly magnetized in these oscillations and can experience Cerenkov resonance in wave-particle interactions such that

$$
\frac{\omega}{k_{\|}}=v_{\| e},
$$

where $v_{\| e}$ is the electron velocity and $k_{\|}$is the wave number, both parallel to the magnetic field. At the same time, protons in these oscillations do not feel the presence of the magnetic field and can oscillate freely across the field lines. The condition for wave-particle interactions in this case is Cerenkov resonance between protons with velocity $v_{i}$ and waves with phase velocities perpendicular to the magnetic field such that

$$
\frac{\omega}{k_{\perp}}=v_{i}
$$

Since lower-hybrid waves have $k_{\|} \ll k_{\perp}$, these waves can be in simultaneous Cerenkov resonance with both fast electrons and slow protons transferring energy from one component to another. This feature of the lower-hybrid waves determines their special role in space physics. There are many space environments in which the energy coupling between the different plasma components is carried out by lowerhybrid waves, one such example is in the auroral plasma, in which the lower-hybrid waves are excited by the flux of precipitating electrons and leads to strong cross-field ion heating (Lynch et al. 1996; Lynch et al. 1999) leading to the formation of ion conics (Chang and Coppi 1981), and ion heating (Retterer et al. 1986; Chang et al. 1988). Alternately, ion horseshoe distributions can also lead to strongly growing lower-hybrid waves that can accelerate electrons parallel to the magnetic field (Bingham et al. 1984; Bingham et al. 1988; Cairns et al. 1995; Bingham et al. 2001). The formation of lower-hybrid solitary structures (LHSS) are also observed in the auroral region of space plasmas (Labelle et al. 1986; Schuck et al. 1998; Schuck et al. 2003).

The physics of lower-hybrid waves can be explained in a simple way, they are ion plasma oscillations in the dielectric medium with the dielectric constant created by 
the motion of the magnetized electrons. To obtain the dielectric constant we shall start with the equation of electron motion across the magnetic field given by

$$
m_{e} \frac{\partial \mathbf{v}_{e}}{\partial t}=-e \mathbf{E}-\frac{e}{c} \mathbf{v}_{e} \times \mathbf{B} .
$$

By treating the term on the left-hand side for the slow oscillations as the small perturbation, we can write the following equation for the electron velocity

$$
\mathbf{v}_{e}=\frac{c}{B^{2}} \mathbf{E} \times \mathbf{B}-\frac{m_{e} c^{2}}{e B^{2}} \frac{\partial \mathbf{E}}{\partial t} .
$$

The first term on the right-hand side corresponds to the incompressible $\mathbf{E} \times$ $\mathbf{B}$ drift $\left(\nabla \cdot \mathbf{v}_{e}=0\right.$, since for electrostatic oscillations $\left.\nabla \times \mathbf{E}=0\right)$, the second term is a small $\sim 0\left(\frac{\omega}{\omega_{c e}}\right)$ correction to the electron velocity corresponding to the polarization drift in the direction of the electric field with $\omega_{c e}=e B / m_{e} c$, the electron cyclotron frequency. The corresponding density perturbation can be found from the continuity equation

$$
\frac{\partial n_{e}}{\partial t}=-n_{o} \operatorname{div} \mathbf{v}_{e}=\frac{n_{o} m_{e} c^{2}}{e B^{2}} \operatorname{div} \frac{\partial \mathbf{E}}{\partial t},
$$

or

$$
n_{e}=\frac{n_{o} m_{e} c^{2}}{e B^{2}} \operatorname{div} \mathbf{E},
$$

and then Poisson's equation can be written as

$$
\operatorname{div} \mathbf{E}=-4 \pi e\left(n_{e}-n_{i}\right),
$$

or

$$
\operatorname{div}\left(\epsilon_{e} \mathbf{E}\right)=4 \pi e n_{i},
$$

where $\epsilon_{e}=1+\frac{4 \pi n_{o} m_{e} c^{2}}{B^{2}}=1+\frac{\omega_{p e}^{2}}{\omega_{c e}^{2}}$ is the dielectric constant created by the polarization of the electron fluid, and $\omega_{p e}$ is the electron plasma frequency $\left(\omega_{p e}=\sqrt{\frac{4 \pi n_{e} e^{2}}{m_{e}}}\right)$. The frequency of the plasma oscillations in a plasma medium with dielectric constant $\epsilon_{e}$ can be written as

$$
\omega=\frac{\left(\omega_{p p}^{2}+\omega_{p e}^{2} \frac{k_{\| \frac{1}{2}}^{2}}{k^{2}}\right)}{\sqrt{\epsilon_{e}}}=\frac{\left(\omega_{p p}^{2}+\omega_{p e}^{2} \frac{k_{\|}^{2}}{k^{2}}\right)^{\frac{1}{2}}}{\left(1+\frac{\omega_{p e}^{2}}{\omega_{c e}^{2}}\right)^{\frac{1}{2}}},
$$

where

$$
\omega_{p p}=\sqrt{\frac{4 \pi n_{o} e^{2}}{m_{p}}}
$$

is the proton plasma frequency. In (7) we take into account that in parallel with ion plasma oscillations electron plasma oscillations are also possible. However, since electrons are magnetized and can move only along the field lines, their plasma frequency is equal to $\omega_{p e} \frac{k_{\|}}{k}$. In many regions of space plasmas the condition $\omega_{p e} \gg \omega_{c e}$ is usually fulfilled, then assuming $\frac{k_{\|}}{k} \ll \sqrt{\frac{m_{e}}{m_{p}}}$, it is easy to verify from the above expression for $\omega$ that the frequency of the lower-hybrid oscillations is equal to the 
geometrical mean of the electron and proton gyrofrequencies

$$
\omega_{l h}=\sqrt{\omega_{c e} \omega_{c p}} .
$$

It is now widely believed that the low-frequency waves observed in the auroral regions are lower-hybrid oscillations excited by the flux of precipitating electrons (Maggs 1976) or ion horseshoe distributions (Cairns et al. 1995). The typical distribution function of the precipitating electrons (Ackerson and Frank 1972) are energetic tails. There are two mechanisms by which these electrons can drive lowerhybrid waves: (a) the "bump on tail" instability (Chang and Coppi 1981), and (b) the "fan" instability due to the anisotropy of the electron distribution (Omelchenko et al. 1994). The 'fan' instability is also known as the anomalous Doppler resonance instability or anomalous cyclotron resonance instability. This instability is important in understanding runaway in tokamaks, and has applications in the cyclotron maser instability (Bingham and Cairns 2001). The growth rate for these waves can be written as

$$
\gamma=\frac{\pi \alpha^{3} \omega_{c e}^{2}}{4 n_{o} k}\left[f_{e}\left(\frac{\omega+\omega_{c e}}{k_{\|}}\right)-f_{e}\left(\frac{\omega-\omega_{c e}}{k_{\|}}\right)+\frac{2 \omega_{c e} k_{\|}}{k^{2}} \frac{\partial f_{e}}{\partial v_{\|}}\right]
$$

where $\alpha=\frac{1}{\sqrt{1+\left(\omega_{p e}^{2} / \omega_{c e}^{2}\right)}}$. The last term in this expression describes wave excitation due to Cerenkov resonance between electrons and waves, $\omega=k_{\|} v_{e}$. In this case the instability is possible due to the presence of the "bump" on the electron distribution function $\partial f_{e} / \partial v_{\|}>0$. The first two terms in the expression for the growth rate are due to the cyclotron resonance between electrons and waves, $\omega-k_{\|} v_{e}= \pm \omega_{c e}$. Here "+" corresponds to the normal cyclotron resonance with waves, "-" to the anomalous cyclotron resonance. Since $f_{e}\left(v_{\|}\right) \rightarrow 0$ for $v_{\|}<0$, the input from the first term (anomalous cyclotron resonance) is dominant leading to instability.

From the consideration of the electron distribution functions that form asymmetric high-energy tails, it follows that the free energy source for wave excitation by cyclotron resonance is essentially more significant than by Cerenkov resonance. In the latter case the driver for the instability is only a "bump" on the velocity distribution, while in the case of the cyclotron resonance, the instability can be driven by the energetic tail of the electron distribution function.

It is easy to follow the evolution of the electron distribution due to the instability driven by cyclotron resonance. For this purpose it is convenient to use the quantum mechanical analogy: Electron energy in the transverse motion is quantized with the energy difference between levels equal to $\hbar \omega_{c e}$. Then the change in transverse energy as a result of the transition between levels can be represented as

$$
m_{e} v_{\perp} \Delta v_{\perp}=n \hbar \omega_{c e},
$$

where $n$ is integer. Positive value of $n=+1$ corresponds to the case where, due to wave excitation, the transverse energy increases (the energy source is in the longitudinal motion - anomalous cyclotron resonance). Negative value $n=-1$ corresponds to the normal cyclotron resonance, when the energy source for wave excitation is in the transverse motion. Using the condition for cyclotron resonance $k_{\|} v_{\|}=\omega-n \omega_{c e}$, it is possible to rewrite the rate of change of the transverse energy to the rate of change of the longitudinal energy in the following way,

$$
\frac{m_{e} v_{\perp} \Delta v_{\perp}}{m_{e} v_{\|} \Delta v_{\|}}=-1+\frac{\omega}{k_{\|} v_{\|}},
$$


where we also used the momentum conservation law

$$
m_{e} \Delta v_{\|}=\hbar k_{\|} .
$$

Integrating (11) we can write the following equation for the particle trajectories along which they move (diffusion lines) interacting with waves satisfying the condition of cyclotron resonance

$$
w=v_{\perp}^{2}+v_{\|}^{2}-2 \int \frac{\omega}{k_{\|}} d v_{\|}=\text {const. }
$$

Since the frequency of lower-hybrid waves satisfy $\omega \ll \omega_{c e}$, the last term in (12) is small, and the diffusion lines are close to circles in velocity space. Due to the pitch angle diffusion created by waves the initial field-aligned distribution of the precipitating electrons opens like a fan (the origin of the term fan instability for the instability under consideration). This fan instability does not require an electron beam or positive slope in the electron distribution function, but only an asymmetric high-energy tail on one side of the distribution function.

The self-consistent quasilinear system of equations describing wave excitation with the evolution of the electron distribution can be written as (Omelchenko et al. 1994)

$$
\begin{gathered}
\frac{\partial\left|E_{k}\right|^{2}}{\partial t}=\frac{4 \pi^{2} e^{2}}{m_{e} k_{\|}\left|k_{\|}\right|} \frac{\omega^{3}}{\omega_{p e}^{2}} \int d \mathbf{v}_{\perp} J_{1}^{2}\left(\frac{k_{\perp} v_{\perp}}{\omega_{c e}}\right) \frac{\partial f_{e}}{\partial v_{\|}}\left|E_{k}\right|^{2}, \\
\frac{\partial f_{e}}{\partial t}=\frac{e^{2}}{2 \pi m_{e}^{2}} \frac{\partial}{\partial v_{\|}} \int d \mathbf{k}_{\perp}\left|E_{k}\right|^{2} \frac{k_{\|}^{2}}{k^{2}} \frac{J_{1}^{2}\left(\frac{k_{\perp} v_{\perp}}{\omega_{c e}}\right)}{\left|v_{\|}-\frac{\partial \omega}{\partial k_{\|}}\right|_{\left(\omega+\omega_{c e}=k_{\|} v_{\|}\right)}} \frac{\partial f_{e}}{\partial v_{\|}},
\end{gathered}
$$

with

$$
\omega+\omega_{c e}=k_{\|} v_{\|}
$$

The derivatives over $v_{\|}$on the right-hand side of these equations are calculated along the diffusion lines, $w=$ constant, and $\left|E_{k}\right|^{2}$ is the spectral density of the square of the wave electric field.

Pitch angle diffusion by waves results in the formation of a "plateau" along the diffusion lines $w=$ const., $f_{e}=f_{e}(w)$. The height of the plateau can be found from the conservation of the number of particles diffusing along these lines

$$
f_{e}^{\infty}=\frac{1}{\sqrt{w}} \int_{v_{1}}^{\sqrt{w}} d v_{\|} f^{o}\left(w-v_{\|}^{2}, v_{\|}\right),
$$

where $v_{1}$ is the minimum value of the resonant phase velocity on the diffusion line corresponding to the wave propagation along the magnetic field $v_{1}=\frac{\omega_{c e}}{k}(1+\alpha)$. For the initial plateau-like distribution of the precipitating electrons in $v_{\|}$,

$$
f_{e}\left(v_{\|}\right)=\left\{\begin{array}{l}
\frac{n_{T e}}{v_{o}}, \text { for } 0<v_{\|}<v_{o} \\
0, \text { for } v_{\|}<0, v_{\|}<v_{o} .
\end{array}\right.
$$

The final distribution has the form

and

$$
f_{e}^{\infty}(w)=\frac{n_{T e}}{\sqrt{w}} \text { for } w<v_{o}^{2},
$$

$$
f_{e}^{\infty}(w)=0 \text { for } w>v_{o}^{2}
$$


Assuming that the electric field spectral density is monochromatic in $k_{\perp}\left(\left|E_{k}\right|^{2} \sim\right.$ $\left.\delta\left(k_{\perp}-k_{o}\right)\right)$, it is possible to write the energy integral of the quasi linear (13) and (14) expressing the electric field spectral density through the change of the electron distribution function as

$$
\left|E_{k}\right|^{2}=\frac{16 \pi^{4} \alpha^{3} m_{e} \omega_{c e}^{4}}{\omega_{p e}^{2} k_{o}^{4}} \sin ^{3} \theta \delta\left(k_{\perp}-k_{o}\right) \int_{v_{1}}^{v_{\|}(\theta)} d v_{\|} \int_{o}^{\infty} v_{\perp} d v_{\perp}\left[f_{e}^{\infty}(w)-f_{e}^{o}\left(v_{\perp}, v_{\|}\right)\right],
$$

using the resonance condition $\omega-k_{\|} v_{\|}=-\omega_{c e}$ and the dispersion equation for $\frac{k_{\|}}{k}>\sqrt{\frac{m_{e}}{m_{p}}}$, as $\omega=\omega_{c e} \alpha \frac{k_{\|}}{k}$, it is easy to express the resonant velocity $v_{\|}(\theta)$ through the propagation angle $\theta$ as

$$
v_{\|}(\theta)=\frac{\omega_{c e}}{k_{o}}\left(\alpha+\frac{1}{\cos \theta}\right), \cos \theta=\frac{k_{\|}}{k} .
$$

Assuming that the precipitating electrons initially had a plateau-like distribution in $v_{\|}$, defined by (16), it is easy to evaluate the spectral density of the wave electric field

$$
\left|E_{k}\right|^{2}=\frac{8 \pi^{3} \alpha^{3} n_{T e} m_{e} \omega_{c e}^{4}}{\omega_{p e}^{2} k_{o}^{4} \Gamma} \delta\left(k_{\perp}-k_{o}\right)(1-\cos \theta) \frac{\sin ^{3} \theta}{\cos ^{3} \theta} \ln \left(\frac{\Gamma-1-\alpha}{1-\cos \theta} \cos \theta\right),
$$

where the notation $\Gamma=\frac{k_{o} v_{o}}{\omega_{c e}}=\frac{v_{o}}{v_{1}}(1+\alpha) \gg 1$ is used. The cutoff of the velocity distribution (16) at its upper boundary leads to the cutoff of the spectral density from the side of the smaller $\cos \theta$ (or $\omega$ ). The minimum value of $\cos \theta=\alpha \frac{\omega}{\omega_{c e}}$ for the excited waves is defined by the relation $\cos \theta_{o}=\frac{1}{\Gamma-\alpha}$. For all reasonable values of $\Gamma(\Gamma \geq 10)$ the maximum of $\left|E_{k}\right|^{2}$ is such that the frequency corresponding to that maximum shifts into the region $\omega \leq 0.1 \alpha \omega_{c e}$ closer to the lower-hybrid frequency (Omelchenko et al. 1994). Therefore the maximum of the wave spectrum must be in the frequency interval $\omega \leq 0.1 \alpha \omega_{c e}$ closer to the lower-hybrid waves. To compare analytical results with the auroral observations from Alouette 2 (Barrington et al. 1971), we calculated the spectral density of the power flux

$$
F(\omega)=\int \frac{d k_{\perp} k_{\perp}}{2 \pi} \frac{\partial \omega}{\partial k_{\|}} \frac{\left|E_{k}\right|^{2}}{4 \pi}\left(1+\frac{\omega_{p e}^{2}}{\omega_{c e}^{2}}\right)=\int d k_{\perp} \omega \frac{\left|E_{k}\right|^{2}}{8 \pi^{2}}\left(1+\frac{\omega_{p e}^{2}}{\omega_{c e}^{2}}\right)^{2} .
$$

We used two types of initial velocity distributions for the precipitating electrons: a power-like distribution

$$
f_{e}\left(v_{\|}\right)=\frac{n_{T e} v_{o}}{v_{\|}^{2}}
$$

and a plateau-like distribution determined by (16). Results of the analytical calculations agree well with the observations of Barrington et al. (1971). In spite of the fact that analytical results depend on the form of the initial electron distribution agreement between them and the observations is good. Calculating the total wave energy density excited by the mechanism of the fan instability in the auroral regions and comparing with the electron energy density, we have

$$
W_{\text {Wave }}=\frac{\omega_{p e}^{2}}{\omega_{c e}^{2}} \sum \frac{\left|E_{k}\right|^{2}}{4 \pi} \approx \frac{v_{1}}{v_{o}} W_{e},
$$


where $W_{e}=\int \frac{m_{e} v_{\|}^{2}}{2} f_{e}\left(v_{\|}\right) d v_{\|}$is the electron energy density. Appearance of the small factor $\frac{v_{1}}{v_{o}}$ on the right-hand side of $(20)$ reflects the fact that the electron diffusion lines are close to circles, and the energy change on them is small $\sim \frac{\omega}{\omega_{c e}}$. The wave energy determined from (20) corresponds to the amplitude of the electric field $E \sim(100-300) \frac{m V}{m}$ for reasonable values of the energy flux of the precipitated electrons $J \sim(3-30) \frac{\mathrm{erg}}{\mathrm{cm}^{2} \mathrm{sec} .}$. Such values of electric field in the lower-hybrid oscillations have been usually observed in the auroral ionosphere (Kintner et al. 1992), and they are definitely above the threshold for the modulational instability of lower-hybrid waves derived by Shapiro et al. (1995b).

\section{Acknowledgements}

We would like to thank the Science and Technology Facilities Council's Centre for Fundamental Physics (CfFP) for support. This work was supported by EPSRC Grant EP/G04239X.

\section{References}

Ackersons, K. L. and Frank, L. A. 1972 J. Geophys. Res. 77, 1128.

Barrington, R. E., Hartz, T. R. and Harvey, R. W. 1971 J. Geophys. Res. 76, 5278.

Bingham, R., Bryant, D. A. and Hall, D. S. 1984 Geophy. Res. Lett. 11, 327.

Bingham, R., Bryant, D. A. and Hall, D. S. 1988 Ann. Geophys. 6, 159.

Bingham, R., Shapiro, V. D., Gil'Man, M., Tsytovich, V. N. and de Angelis, U. 1991 Phys. Fluids B 3, 1728.

Bingham, R., Su, J. J., Shapiro, V. and Dawson, J. M. 1996 Phys. Scripta T63, 46.

Bingham, R., Dawson, J. M., Shapiro, V. D., Mendis, D. A. and Kellet, B. J. 1997 Science 275,49 .

Bingham, R., Kellett, B. J., Cairns, R. A., Dendy, R. O. and Shukla, P. K. 1999 Geophys. Res. Lett. 26, 2713.

Bingham, R. and Cairns, R. A. 2000 Phys. Plasmas 7, 3089.

Bingham, R., Cairns, R. A., Dendy, R. O., Shapiro, V. D., Shukla, P. K. and Stenflo, L. 2001 Phys. Chem. Earth (C) 26, 133.

Bingham R., Dawson J. M., Shapiro V. D., 2002, J. Plasma Phys. 68, 161.

Bingham, R., Shapiro, V. D., Shukla, P. K. and Trines, R. 2004a Phys. Scripta T113, 144.

Bingham, R., Kellett, B. J., Bryans, P., Summers, H. P., Torney, M., Shapiro, V. D., Spicer, D. S. and O'Brien, M. 2004b Astrophys. J. 601, 896.

Bingham, R., Quest, K. B., Shapiro, V. D. and Kellett, B. J. 2008 Ann. Geophys. 26, 1829.

Cairns, R. A. 1991 Radio Frequency Heating of Plasmas. Bristol, UK: Adam Hilger.

Cairns, R. A., Mamum, A. A., Bingham, R., Boström, R., Dendy, R. O., Nairn, C. M. C. and Shukla, P. K. 1995 Geophys. Res. Lett. 22, 2709.

Chang, T. and Coppi, B. 1981 Geophys. Res. Lett. 8, 1253.

Chang, T., Crew, G. B. and Retterer, J. M. 1988 Comput. Phys. Commun. 49, 61.

Johnstone, A. D., Coates, A. J., Heath, J., Thomsen, M. F., Wilken, B., Jockers, K., et al. 1987 Astron. Astrophys. 187, 25.

Karney, C. F. F. 1978 Phys. Fluids 21, 1584.

Karney, C. F. F. and Fisch, N. J. 1979 Phys. Fluids 22, 1817.

Kintner, P. M., Vago, J., Chesney, S., Arnoldy, R. L., Lynch, K. A., Pollock, C. J. and Moore, T. E. 1992 Phys. Rev. Lett. 68, 2448.

Labelle, J., Kintner, P. M., Yau, A. W. and Whalen, B. A. 1986 J. Geophys. Res. 91, 7113.

Labelle, J. and Treumann, R. A. 1988 Space Sci. Rev. 47, 175. 
Lembege, B. and Dawson J. M. 1986 Phys. Fluids 29, 821.

Lynch, K. A., Arnoldy, R. L., Kintner, P. M. and Bonnell, J. 1996 Geophys. Res. Lett. 23, 3293.

Lynch, K. A., Arnoldy, R. L., Kintner, P. M., Schuck, P., Bonnell, J. W. and Coffey, V. 1999 J. Geophys. Res. 104, 28515.

Maggs, J. E. 1976 J. Geophys. Res. 81, 1707.

McClements, K. G., Bingham, R., Su, J. J., Dawson, J. M. and Spicer, D. S. 1990 Solar Phys. 130,229 .

Omelchenko, Y. A., Shapiro, V. D., Shevchenko, V. I., Ashour-Abdalla, M. and Schriver, D. 1994 J. Gephys. Res. 99, 5965.

Paschmann, G., Sckopke, N., Bame, S. J. and Gosling, J. T. 1982 Geophys. Res. Lett. 9, 881.

Retterer, J. M., Chang, T. and Jasperse, J. R. 1986 J. Geophys. Res. 91, 1609.

Robinson, P. A. 1997 Rev. Mod. Phys. 69, 507.

Schuck, P. W., Seyler, C. E., Pinçon, J.-L., Bonnell, J. W. and Kintner, P. M. 1998 J. Geophys. Res. 103, 6935.

Schuck, P. W. and Bonnell, J. W. 2003 J. Geophys. Res. 108, 1175.

Shapiro, V. D., Szegö, K., Ride, S. K., Nagy, A. F. and Shevchenko, V. I. 1995a J. Geophys. Res. 100, 21289.

Shapiro, V. D., Soloviev, G. I., Dawson, J. M. and Bingham, R. 1995 Phys. Plasmas 2, 516.

Shapiro, V. D., Bingham, R., Dawson, J. M., Dobe, Z., Kellett, B. J. and Mendis, D. A. 1999 J. Geophys. Res. 104, 2537.

Sotnikov, V. I., Shapiro, V. D. and Shevchenko, V. I. 1980 Sov. Phys. JETP 51, 295.

Trines, R., Bingham, R., Silva, L. O., Mendonça, J. T., Shukla, P. K. and Mori, W. B. 2005 Phys. Rev. Lett. 94, 165002.

Trines, R., Bingham, R., Dunlop, M. W., Vaivads, A., Davies, J. A., Mendonça, J. T., Silva, L. O. and Shukla, P. K. 2007 Phys. Rev. Lett. 99, 205006. 